\title{
CARACTERIZACIÓN BIOFÍSICA DE LOS HÁBITATS DE POBLACIONES NATURALES DE CAMU CAMU Myrciaria dubia (H.B.K) Mc Vaugh
}

\author{
BIOPHYSIC CHARACTERIZATION OF CAMU CAMU Myrciaria dubia (H.B.K) \\ Mc Vaugh NATURAL POPULATIONS HABITATS
}

\section{Agustín Gonzales Coral ${ }^{1}$}

\section{RESUMEN}

Se caracterizaron poblaciones de camu camu: Supay Cocha $73^{\circ} 42^{\prime} 06^{\prime \prime}$ oeste y $04^{\circ} 56^{\prime} 20^{\prime \prime}$ sur, Sahua Cocha $73^{\circ}$ $42^{\prime} 51^{\prime \prime}$ oeste y $04^{\circ} 56^{\prime} 44^{\prime \prime}$ sur, en el río Ucayali; Palmichal y Tapaje $73^{\circ} 13^{\prime} 35^{\prime}$ " oeste y $04^{\circ} 18^{\prime} 40^{\prime}$ " sur en el río Tahuayo.

Las poblaciones, en el año 1999, recibieron inundaciones entre $7.60 \mathrm{~m}$ a $10.30 \mathrm{~m}$ para el caso de Supay Cocha. Para Sahua Cocha entre $7.50 \mathrm{~m}$ a $9.70 \mathrm{~m}$. Palmichal entre $7.30 \mathrm{~m}$ y $12.10 \mathrm{~m}$, y Tapaje entre $5.60 \mathrm{~m}$ y $11.50 \mathrm{~m}$. En el año 2000, Supay Cocha recibió inundación entre $8.20 \mathrm{~m}$ a $8.30 \mathrm{~m}$, Sahua Cocha entre $7.20 \mathrm{~m}$ y $8.25 \mathrm{~m}$, Palmichal entre $5.00 \mathrm{~m}$ a $9.00 \mathrm{~m}$, y Tapaje entre $5.30 \mathrm{~m}$ y $5.80 \mathrm{~m}$. Los suelos son predominantemente franco-arcillo-arenoso y arcillosos, con acidez muy alta, con concentraciones de nitrógeno, materia orgánica, fósforo, potasio, calcio, magnesio y potasio cambiable de mediana para alta.

La densidad de plantas por hectárea, a un metro de altura del suelo, fue desde 27800 hasta 46275 ramas; además plantas atípicas que van desde el 2657 a 12350; la producción por hectárea de 9125 a $17450 \mathrm{~kg}$.

Sus registros en promedio fueron: largo $2.16 \mathrm{~cm}$, diámetro $2.30 \mathrm{~cm}$, peso $6.81 \mathrm{~g}$, semillas por frutos 2.82 , peso cáscara $1.31 \mathrm{~g}$, peso semilla $1.45 \mathrm{~g}$, y pulpa $4.06 \mathrm{~g}$. La composición porcentual de frutos fue: cáscara $19 \%$, semilla $21 \%$ y pulpa $60 \%$.

Palabras clave: Myrciaria dubia, caracterización, supay, sahua, palmichal, tapaje, Amazonía peruana.

\begin{abstract}
Populations of camu camu were characterized in: Supay Cocha ( $73^{\circ} 42^{\prime} 06^{\prime \prime}$ west and $04^{\circ} 56^{\prime} 20^{\prime \prime}$ South), Sahua Cocha ( $73^{\circ} 42^{\prime} 51^{\prime \prime}$ "west and $04^{\circ} 56^{\prime} 44^{\prime \prime}$ South), in the Ucayali river. Palmichal and Tapaje ( $73^{\circ} 13^{\prime} 35^{\prime \prime}$ west and $04^{\circ} 18^{\prime} 40^{\prime}$ 'South), in the Tahuayo river. The populations in year 1999 were flooded between $7.60 \mathrm{~m}$ to $10.30 \mathrm{~m}$ in the Supay Cocha; between $7.50 \mathrm{~m}$ to $9.70 \mathrm{~m}$ in the Sahua Cocha; in Palmichal between $7.30 \mathrm{~m}$ and $12.10 \mathrm{~m}$; in Tapaje between $5.60 \mathrm{~m}$ and $11.50 \mathrm{~m}$. In the year 2000, Supay Cocha flooded $8.20 \mathrm{~m}$ to $8.30 \mathrm{~m}$; Sahua Cocha between $7.20 \mathrm{~m}$ and $8.25 \mathrm{~m}$; Palmichal between $5.00 \mathrm{~m}$ and $9.00 \mathrm{~m}$; and Tapaje between $5.30 \mathrm{~m}$ and $5.80 \mathrm{~m}$. The grounds are predominantly franc-argillaceous-sandy argillaceous, with very high acidity, concentrations of Nitrogen, Organic Matter, Phosphorus, Potassium, Calcium, Magnesium and changeable Potassium of medium for discharge. The density of plants by hectare, a meter-high from the ground, ranged from 27800 to 46275 branches. Additionally, atypical plants were from 2657 to 12350; the production by hectare was between 9125 to $17450 \mathrm{~kg}$. Average values were: length $2.16 \mathrm{~cm}$, diameter $2.30 \mathrm{~cm}$, weight $6.81 \mathrm{~g}$, seeds by fruits 2.82 , weight rind $1.31 \mathrm{~g}$, weight seed $1.45 \mathrm{~g}$ and 4.06 pulp g. The percentage composition of fruits: rind $19 \%$, seed $21 \%$ and pulp of $60 \%$.
\end{abstract}

Keywords: Myrciaria dubia, characterization, supay, sahua, palmichal, tapaje, Peruvian amazon.

1 Instituto de Investigaciones de la Amazonía Peruana, Programa de Biodiversidad. Correo electrónico: agonzales@iiap.org.pe 


\section{INTRODUCCIÓN}

El uso, manejo y conservación de los recursos son actividades muy importantes para la inclusión de nuevos productos de la biodiversidad en la economía del poblador amazónico. En los años 90 la especie Myrciaria dubia «camu camu», toma singular importancia por la demanda en los mercados internacionales, lo que trajo como consecuencia la preocupación de muchas instituciones en realizar investigaciones orientadas a un mayor conocimiento de estas áreas y facilitar un manejo adecuado.

El gran interés por esta fruta se debe especialmente a su gran contenido de ácido ascórbico en los frutos (1230 $\mathrm{mg} / 100 \mathrm{~g}$ a $2089 \mathrm{mg} / 100 \mathrm{~g}$ de pulpa fresca) (Pinedo et al, 2001), característica de suma importancia en la industria local, nacional e internacional.

La conservación de la especie en su medio natural es muy valiosa, toda vez que el abastecimiento de los mercados locales, nacionales e internacionales en su gran mayoría procede de poblaciones naturales, que se encuentran a lo largo de la Amazonía; sin embargo, la sobreexplotación de la especie, por la demanda creciente de los frutos, pone en muchos casos en riesgo la conservación de la especie (BIODAMAZ, 2004).

Las observaciones periódicas que se vienen realizando año tras año indican preliminarmente la reducción progresiva de las poblaciones naturales, siendo necesaria la investigación de las causas de este fenómeno.

\section{MATERIAL Y MÉTODO}

Los estudios de caracterización biofísica de las poblaciones naturales de camu camu Myrciaria dubia (H.B.K) Mc Vaugh, se llevaron a cabo entre los años 1999 y 2000, en cuatro localidades: Supay Cocha $73^{\circ} 42^{\prime}$ '06" longitud oeste y $04^{\circ} 56^{\prime} 20^{\prime \prime}$ latitud Sur y Sahua Cocha $73^{\circ} 42^{\prime}$ '51" longitud oeste y $04^{\circ} 56^{\prime} 44^{\prime \prime}$ latitud sur, ubicado a la margen derecha del río Ucayali a $6 \mathrm{~km}$ de distancia de la ciudad de Jenaro Herrera, en la provincia de Requena; Palmichal y Tapaje: $73^{\circ} 13^{\prime} 35^{\prime}$ " longitud oeste y $04^{\circ} 18^{\prime} 40^{\prime \prime}$ latitud Sur, ubicado a la margen derecha del río Tahuayo, a $2 \mathrm{~km}$ de distancia del caserío El Chino, en el distrito de Fernando Lores, provincia de Maynas, departamento de Loreto, Perú.

Las caracterizaciones de los cuerpos de agua que inundan anualmente a las poblaciones naturales, se realizaron en los meses de máxima inundación: Supay Cocha el día 24 de marzo de 1999, Sahua Cocha el 25 de marzo, Palmichal y Tapaje el 13 de abril de 1999. En el año 2000 las mediciones se realizaron: 22 de marzo para Supay Cocha y Sahua Cocha. El 30 de marzo para Palmichal y Tapaje, efectuándose paralelamente los muestreos de agua.

Los muestreos de los suelos donde se desarrollan los rodales naturales se llevaron a cabo en el mes de agosto de 1999 y setiembre del 2000, cuando gran parte de las poblaciones naturales se encontraban sin influencia de agua. En cada población natural se realizaron muestreos de suelos a dos profundidades: $0.01 \mathrm{~m}$ a $0.15 \mathrm{~m}$ y de $0.16 \mathrm{~m}$ a $0.30 \mathrm{~m}$.

Las muestras de aguas y suelos fueron analizadas en los Laboratorios de Aguas y Suelos de la Facultad de Ingeniería Química de la Universidad Nacional de la Amazonía Peruana.

Para el Inventario Poblacional de las poblaciones naturales, se realizaron mediciones a lo largo y ancho de los rodales naturales con la finalidad de tener una aproximación del área. Posteriormente, se ubicaron parcelas de 100 $\mathrm{m}^{2}$, donde se efectuaron los conteos de ramas a un metro de altura del suelo, distribuidas en bases diamétricas de 0.1 a $2.5 \mathrm{~cm} ; 2.6$ a $5.0 \mathrm{~cm}$ y mayores de $5.1 \mathrm{~cm}$, especies atípicas, entre otras.

En cada una de las parcelas ubicadas se marcaron 10 plantas por cada base diamétrica, en las que se realizaron las evaluaciones de la producción.

Al momento de la cosecha se registraron las mediciones y pesadas de los frutos, así como los cálculos de la composición porcentual del fruto. 


\section{RESULTADOS Y DISCUSIÓN}

\subsection{Caracterización limnológica de las aguas de inundación y suelos de las poblaciones naturales}

Niveles de Inundación. Las poblaciones naturales de camu camu anualmente reciben mayor o menor grado de inundaciones de las aguas de los ríos aledaños. Existe la posibilidad de incrementar la calidad de los suelos cuando la inundación es mayor, debido a que se produce el arrastre de materia orgánica (vegetal o animal) de las partes más altas de las cuencas, sedimentando en las partes más bajas.

- Supay Cocha: Los máximos niveles de inundación se registraron entre los meses de marzo y abril. Las mediciones de profundidad, en el año 1999, registraron a los alrededores entre $7.60 \mathrm{~m}, 7.70 \mathrm{~m}, 8.00 \mathrm{~m}$ y 7.90 $\mathrm{m}$, y en la parte central de la cocha $10.30 \mathrm{~m}$. En esta oportunidad se pudo observar pocas copas de las plantas que sobrepasaban el nivel de agua hasta en $60 \mathrm{~cm}$. En el año 2000, los registros de niveles de inundación fluctuaron entre $8.20 \mathrm{~m}$ y $8.30 \mathrm{~m}$.

- Sahua Cocha: Los máximos niveles de inundación se registraron en los meses de marzo y abril. Las mediciones de profundidad, en el año 1999, registraron en los alrededores profundidades de $7.80 \mathrm{~m}, 7.70 \mathrm{~m}, 7.80 \mathrm{~m}, 7.50$ $\mathrm{m}, 7.80 \mathrm{~m}$, y en la parte central de la cocha $9.70 \mathrm{~m}$. Pocas plantas lograron sobrepasar estas profundidades, mostrando la parte superior de las copas hasta en $45 \mathrm{~cm}$. En el año 2000, los registros de niveles de inundación se tomaron en el mes de marzo con mediciones entre $7.20 \mathrm{~m}$ y $8.25 \mathrm{~m}$.

- Palmichal: Los registros de máxima inundación se realizaron en abril de 1999. Los datos registrados corresponden a mediciones en forma transversal de la margen derecha hacia la izquierda, reportando profundidades de $7.30 \mathrm{~m}, 7.50 \mathrm{~m}, 9.40 \mathrm{~m}, 12.10 \mathrm{~m}, 8.10 \mathrm{~m}$, y $7.90 \mathrm{~m}$. En el año 2000, los registros de niveles de inundación se tomaron el 30 de marzo con mediciones entre $5.00 \mathrm{~m}$ y $9.00 \mathrm{~m}$.

- Tapaje: Los registros de las profundidades se realizaron en el mes de abril de 1999, con profundidades entre $5.60 \mathrm{~m}$ y $11.80 \mathrm{~m}$. En el año 2000, los registros de niveles de inundación se efectuaron en el mes de marzo, con mediciones entre $5.30 \mathrm{~m}$ y $5.80 \mathrm{~m}$.

\section{Limnología de las aguas que inundan las poblaciones naturales}

De acuerdo al Anexo 1 los parámetros limnológicos medidos y evaluados se reportan como sigue:

- Temperatura: La temperatura media de los cuerpos de agua entre los años 1999 y 2000 fue de $27.8^{\circ} \mathrm{C}$ y 26.0 ${ }^{\circ} \mathrm{C}$ para Supay Cocha; $27.9^{\circ} \mathrm{C}$ y $26.0^{\circ} \mathrm{C}$ para Sahua Cocha; $27.8^{\circ} \mathrm{C}$ y $26.50^{\circ} \mathrm{C}$ para Palmichal y Tapaje; sin mayores diferencias de la temperatura tomada en el año 1982, que fue de $26^{\circ} \mathrm{C}$ (Canepa, 1982). La temperatura es un factor que puede determinar que la actividad microbiana en el agua, favorezca la descomposición de la materia orgánica y sus derivados $\mathrm{y}$, por consiguiente, los aportes de nutrientes al suelo, donde se desarrollan las poblaciones naturales de camu camu.

- $\quad$ pH: Los reportes registrados entre los años 1999 y 2000 para Supay Cocha fueron de 6.85 y 6.45 unidades, para Sahua Cocha 6.95 y 6.56 unidades, y para Palmichal y Tapaje 6.85 y 6.42 unidades. Los registros superiores indica mayor cantidad de compuestos alcalinos, debido a que posiblemente esta agua recibe parte de agua de inundación, que arrastra mayores cantidades de sólidos en suspensión, los que van a sedimentarse y repercutir en la calidad de los suelos (IIAP, 1987).

- Turbidez (FTU): Relacionado con la cantidad de sólidos en suspensión como: calcio, magnesio, fosfatos, nitratos, nitritos, sulfatos, entre otros, que van a sedimentarse en el suelo. En el cuadro del Anexo 2 podemos observar que los registros en Supay Cocha sufren un gran descenso en el año 2000 respecto al año 1999, por el momento y la época de muestreo (3 meses después de la máxima inundación). Ocurre lo mismo en Sahua Cocha; en Palmichal y Tapaje muestra un descenso considerable para el año 2000, resultado de la poca inundación recibida. 
- Oxígeno disuelto (mg/l): Las concentraciones de oxígeno disuelto, observado en los dos años y en las tres poblaciones, no tuvieron mayores diferencias (Anexo 2). Esta característica es importante porque a mayor concentración la acción microbiana es mayor, acelerándose los procesos de óxido reducción, lo que va a permitir la descomposición más rápida de los desechos orgánicos en el agua.

- Alcalinidad total $\left(\mathbf{C a C O}_{3}\right)(\mathbf{m g} / \mathbf{l})$ : La alcalinidad está muy relacionada con el $\mathrm{pH}$ en los cuerpos de agua. Los datos del Anexo 2 señalan resultados variados por cada año y por cada población. Estos parámetros son muy importantes porque cuanto mayor sean, indican mayores compuestos alcalinos disueltos en el agua, debido posiblemente a la influencia de las aguas de inundación que arrastran cantidades considerables de sólidos en suspensión, los cuales van a sedimentarse en las partes más bajas de los lagos, mejorando la calidad de los suelos.

- Compuestos nitrogenados: Los resultados del Anexo 2 muestran la presencia de adecuadas cantidades de compuestos nitrogenados para Supay Cocha, Sahua Cocha y Palmichal y Tapaje, respectivamente. La presencia de compuestos nitrogenados en las aguas es el resultado de la descomposición de la materia orgánica, ya sea de origen animal o vegetal, excreción de animales, actividad microbiana como los nitrosomonas y nitrobacter. También incluye la liberación del fondo de los suelos por la acción de las corrientes de las aguas, o por animales ventómicos que se encuentran removiendo los sedimentos, o por insectos, entre otros. Los resultados tienen mínimas diferencias con los reportes de Canepa (1982) realizados en Supay Cocha y Sahua Cocha.

\section{Edafología de los suelos de las poblaciones naturales de camu camu}

De acuerdo al Anexo 2, los análisis de suelos realizados durante 1999 y 2000 reportan para Supay Cocha textura arcillosa, mientras que para Sahua Cocha en 1999 la predominancia de arenas es hasta 70\%, debido posiblemente al ingreso de las aguas del río Ucayali en las poblaciones naturales, cambiando en el 2000, cuando la predominancia fue arcilla y limo. En las poblaciones de Palmichal y Tapaje no se observaron mayores variaciones, manteniéndose con una estructura arcillosa. La reacción de los suelos en Supay Cocha y Sahua Cocha, en 1999, se presenta muy ácida, con pequeñas variaciones favorables en el 2000. En las poblaciones de Palmichal y Tapaje mantienen su calidad de muy ácido y no muestran variaciones considerables en los dos años.

Los porcentajes de nitrógeno en las poblaciones evaluadas se incrementan de medios, en el 1999, a altos en el año 2000. La materia orgánica en las poblaciones de Supay Cocha y Sahua Cocha se mantienen en porcentajes medios, y en Palmichal y Tapaje se encontraban en altos porcentajes.

En lo que respecta al elemento fósforo, en la población Supay Cocha, en el año 1999, se encontraba en concentraciones medias, incrementándose en el año 2000 a concentraciones altas; en Sahua Cocha se mantienen en concentraciones altas durante los dos años. En Palmichal, en 1999, se encuentran en concentraciones medias, aumentando a concentraciones altas en el 2000. La población Tapaje en 1999 se encontraba en concentraciones bajas, incrementándose en el 2000 a concentraciones altas. En la tabla 2 se nota incremento de un año para otro, posiblemente porque mejora la estructura del suelo, básicamente en los componentes arena, limo y arcilla, ya que este elemento se presenta en mayores cantidades en suelos orientados a francos, y no así en los arenosos (Rodríguez, 1992).

Con respecto al $\mathrm{K}_{2} \mathrm{O}$ como fuente de $\mathrm{K}$, las concentraciones en las poblaciones Supay Cocha y Sahua cocha en los dos años de evaluación se encontraban en concentraciones altas. Mientras que en las poblaciones Palmichal y Tapaje las concentraciones medias registradas en 1999 son incrementadas a concentraciones altas en el año 2000. Las diferencias numéricas de un año a otro están relacionadas principalmente con la metodología del análisis de suelos (Método tetrafenilborato), dado que en 1999 se realizaron cinco muestras sin pasar por el proceso de filtración, y el año 2000 se efectuaron previo filtrado de las muestras.

El K cambiable (meq/100 gr) en las poblaciones de Supay Cocha, Sahua Cocha y Palmichal se encontraba en concentraciones altas durante los años 1999 y 2000; sin embargo, la población Tapaje baja su concentración de alto a medio en el año 2000. 
Las evaluaciones reportan altas concentraciones de calcio cambiable (meq/100 gr) en los suelos de las poblaciones de Supay Cocha y Sahua Cocha durante el período 1999 - 2000, mientras que en las poblaciones Palmichal y Tapaje en 1999 se encontraban en concentraciones medias, incrementándose a concentraciones altas en el 2000.

En los años 1999 y 2000 las concentraciones de magnesio cambiable (meq/100 gr) en las poblaciones de Supay Cocha y Sahua Cocha se encontraban en concentraciones medias, mientras que en las poblaciones Palmichal y Tapaje, en 1999, se encontraban en concentraciones bajas, incrementándose a concentraciones medias en el 2000

\subsection{Inventario poblacional y evaluación de producción de las poblaciones naturales de camu camu}

\section{Inventario poblacional}

En el Anexo 3 se reportan los resultados del inventario de plantas de camu camu en las poblaciones naturales, donde se pueden observar que:

Durante los años 1999 y 2000 la concentración de la especie en las poblaciones naturales fueron: Para Supay Cocha, a un metro de altura del suelo, se registró una concentración de ramas hasta de $27800 /$ ha, distribuidas en cinco bases diamétricas. La mayor concentración se observó entre los diámetros de 2.00 a $2.99 \mathrm{~cm}$ con 6600 ramas, seguido por los de 3.00 a $3.99 \mathrm{~cm}$ con 5800 ramas, después los mayores de $5 \mathrm{~cm}$ con 5700 , luego las de 4.00 a $4.99 \mathrm{~cm}$ con 5200 ramas y, finalmente, las de 0.01 a 1,99 con 4600 ramas. Peters (1988), en Sahua Cocha, reporta la existencia de 14 especies leñosas, con la predominancia de camu camu con 1231 individuos en $1000 \mathrm{~m}^{2}$, más de 12000 individuos por ha; adiciona que las plantas tienen una profusa ramificación y cuando se incluyen rebrotes llegan hasta $1500 \mathrm{~m}^{2}$.

La población de especies atípicas fue de 2657 individuos, representada hasta por cinco especies. La predominancia del camu camu se pudo observar hasta en $91.00 \%$.

Para Sahua Cocha se registraron concentraciones de ramas hasta de 34975, distribuidas en cinco bases diamétricas. La mayor concentración se observó entre los diámetros de 3.00 a $3.99 \mathrm{~cm}$ con 9400 ramas, seguido por los de 4.00 a $4.99 \mathrm{~cm}$ con 8300 ramas, después de 2.00 a $2.99 \mathrm{~cm}$ con 6800 , luego las mayores de $5 \mathrm{~cm}$ con $5300 \mathrm{y}$, finalmente, las de 0.01 a 1.99 con 5200 ramas. La población de especies atípicas fue de 3850 individuos, representada hasta por 4 especies. La predominancia del camu camu se pudo observar hasta en $90.00 \%$.

Para Palmichal se registraron concentraciones de ramas hasta de 37725 , distribuidas en cinco bases diamétricas. La mayor concentración se observó entre los diámetros de 2.00 a $2.99 \mathrm{~cm}$ con 14000 ramas, seguido por los de 0.01 a $1.99 \mathrm{~cm}$ con 11200 ramas, después de 3.00 a $3.99 \mathrm{~cm}$ con 10 100, luego de 4.00 a 4.99 con 2000, y, finalmente, las mayores de $5 \mathrm{~cm}$ con 500. La población de especies atípicas fue de 12350 individuos, representada hasta por dos especies. La predominancia del camu camu se pudo observar hasta en $75.00 \%$.

Para Tapaje, se registraron concentraciones de ramas hasta de 46275 , distribuidas en cinco bases diamétricas. La mayor concentración se observó entre los diámetros de 2.00 a 2.99 cm y 3.00 a 3.99 con 14300 ramas, seguido por los de 0.01 a $1.99 \mathrm{~cm}$ con 12600 ramas, luego de 4.00 a $4.99 \mathrm{~cm}$ con 5900 , y, finalmente, las mayores de $5 \mathrm{~cm}$ con 900. La población de especies atípicas fue de 5250 individuos, representada hasta por cuatro especies. La predominancia del camu camu se pudo observar hasta en $90.00 \%$.

Las especies atípicas en Supay Cocha y Sahua Cocha son similares, con representación importante de la especie Eugenia inundata fanache, con $77 \%$ y $50 \%$, respectivamente. En Palmichal la especie atípica representativa fue Mabea nitida sabalohuayo, con $99 \%$, y en Tapaje la representatividad recayó en dos especies Stylogyne longifolia acarahuazú panga y Mabea nitida sábalohuayo, con $58 \%$ y $40 \%$, respectivamente (Anexo 5). 


\subsection{Evaluación de producción}

Los Anexos 6 y 7 muestran el rendimiento $\mathrm{kg} / \mathrm{ha}$ de las poblaciones naturales de camu camu. Estos rendimientos son muy variables, dependiendo de ciertos factores como el tiempo en que se mantiene la población fuera del agua, ya que dan oportunidad de cosechar los frutos en los brotes más pequeños y por tiempos más prolongados. Asimismo, se observó que las ramas más productivas están entre las bases diamétricas de $2 \mathrm{~cm}$ a $5 \mathrm{~cm}$ (Peters 1988). La población de Supay Cocha en 1999 reporta una producción de $15700 \mathrm{~kg} / \mathrm{ha}$, mientras que en el año 2000 sufre disminución hasta alcanzar los $11150 \mathrm{~kg}$; En Sahua Cocha, en 1999, la producción fue de $17450 \mathrm{~kg} / \mathrm{ha}$, pero disminuyó significativamente en el año 2000 a $9126 \mathrm{~kg} / \mathrm{ha}$; En la población Palmichal no se muestran mayores diferencias entre los dos años: $13400 \mathrm{~kg} / \mathrm{ha}$ y $13525 \mathrm{~kg} / \mathrm{ha}$, respectivamente. En Tapaje la diferencia no es significativa, reportando el año 1999 un rendimiento de $14350 \mathrm{~kg} /$ ha y en el 2000 se observaron volúmenes de $13750 \mathrm{~kg} / \mathrm{ha}$. De manera general, Flores (1997), reporta que en poblaciones naturales los rendimientos pueden estar entre 9500 y $12700 \mathrm{~kg} / \mathrm{ha} / \mathrm{año}$.

Las mediciones biométricas de los frutos reportan promedios variables con diferencias poco significativas (Anexo 7), el largo del fruto de $2.28 \mathrm{~cm}$, diámetro o ancho de $2.43 \mathrm{~cm}$. Mediciones promedios reportadas por Flores (1997): peso fruto $7.69 \mathrm{~g}$, número de semillas de 2.92, peso de semilla de $1.47 \mathrm{~g}$ peso de cáscara de $1.52 \mathrm{~g}, \mathrm{y}$, finalmente, peso de pulpa de $4.70 \mathrm{~g}$. La composición porcentual de los componentes del fruto son: cáscara $20 \%$, semilla $19 \%$ y pulpa de 61\%, existiendo mucha variabilidad en estos caracteres (Flores, 1997) (Villachica, 1996).

\section{BIBLIOGRAFÍA}

CÁNEPA, LA SERNA, R. 1982. Estudio Bioecológico del sábalo cola roja Brycon erythropterum en el Sistema de Lagunas Supay y Aledaños Jenaro Herrera, Requena. U.N. Federico Villarreal. Lima. Perú. Pág. 5-14.

FLORES, P. S. 1997. Cultivo de frutales nativos amazónicos. Manual para el extensionista. Tratado de Cooperación Amazónica. Pág. 55-62

PETERS, C. M.; VÁSQUEZ M. 1988. Estudios Ecológicos de camu camu (Myrciaria dubia) producción de frutos en poblaciones naturales. In Folia Amazónica Vol. 1 Nº 1 P 58-98.

VILLACHICA, H. 1996. Frutales y Hortalizas Promisorias de la Amazonía. Tratado de Cooperación Amazónica. Pág. 75-83.

F. RODRÍGUEZ, G. PAREDES, R. BAZÁN Y J. RAMÍREZ. 1992. Algunas propiedades físico-químicas y mineralógicas de materiales sedimentarios recientes en un complejo de orillares del río Amazonas. Folia Amazónica Vol. 4 No 2. IIAP. Iquitos, Perú. Pág. 5-29. 
Anexo 1. Limnología de las aguas de las poblaciones naturales de camu camu

\begin{tabular}{|c|c|c|c|c|c|c|c|}
\hline \multirow[t]{2}{*}{$\begin{array}{l}\text { Característica } \\
\text { física }\end{array}$} & \multirow[t]{2}{*}{ Unidad } & \multicolumn{2}{|c|}{ Supay } & \multicolumn{2}{|c|}{ Sahua } & \multicolumn{2}{|c|}{$\begin{array}{c}\text { Palmichal y } \\
\text { Tapaje }\end{array}$} \\
\hline & & 1999 & 2000 & 1999 & 2000 & 1999 & 2000 \\
\hline Temperatura & ${ }^{\circ} \mathrm{C}$ & 27.80 & 26.00 & 27.90 & 26.00 & 27.80 & 26.50 \\
\hline $\mathrm{Ph}$ & Unid.Stan. & 6.85 & 6.45 & 6.95 & 6.56 & 6.85 & 6.42 \\
\hline Conductividad & $\mathrm{US} / \mathrm{L}$ & & 101.70 & & 119.00 & 0.07 & 42.00 \\
\hline TDS & $\mathrm{mg} / \mathrm{l}$ & & 51.01 & & 56.16 & & 17.7 \\
\hline Color & Unid. Pt-Co & & 76.00 & & 88.00 & & \\
\hline Turbidez & NTU & 15.50 & 1.87 & 24.00 & 3.92 & 13.5 & 2.20 \\
\hline Oxígeno disuelto & $\mathrm{mg} / \mathrm{l}$ & 7.36 & 6.28 & 9.53 & 7.13 & & 7.3 \\
\hline $\begin{array}{l}\text { Características } \\
\text { químicas }\end{array}$ & & & & & & & \\
\hline Alcalinidad total & $\mathrm{mg} / \mathrm{l}$ & 40.00 & 20.00 & 50.00 & 20.00 & 20.00 & \\
\hline Dureza total & $\mathrm{Mg} / \mathrm{l}$ & & 16.00 & & 34.00 & & \\
\hline Dureza de Calcio & $\mathrm{mg} / \mathrm{l}\left(\mathrm{CaCo}_{3}\right)$ & & 9.27 & & 19.71 & & \\
\hline $\begin{array}{l}\text { Dureza de } \\
\text { Magnesio }\end{array}$ & $\mathrm{mg} / \mathrm{l}\left(\mathrm{CaCo}_{3}\right)$ & & 6.72 & & 14.28 & & \\
\hline Fierro & $\mathrm{mg} / \mathrm{l}$ & & 0.54 & & 0.03 & & \\
\hline Nitratos & $\mathrm{mg} / \mathrm{l}$ & & 3.80 & & 0.48 & & \\
\hline Fósforo & $\mathrm{mg} / \mathrm{l}$ & & 0.03 & & 0.02 & & 0.00 \\
\hline $\mathrm{Ca}+\mathrm{Mg}$ & $\mathrm{meq} / 1$ & & 1.00 & & 1.00 & & 0.20 \\
\hline Estimado de Sodio & meq/1 & & 0.02 & & 0.19 & & 0.22 \\
\hline $\begin{array}{l}\text { Tasa de absorción } \\
\text { de } \mathrm{Na}\end{array}$ & meq/1 & & 0.02 & & 0.26 & & 0.69 \\
\hline Nitrógeno - Nitrato & $\mathrm{mg} / \mathrm{l}$ & 0.40 & 1.00 & 0.30 & 1.00 & 1.00 & 1.00 \\
\hline Nitrógeno - Nitrito & $\mathrm{mg} / \mathrm{l}$ & 0.00 & 0.001 & 0.00 & 0.001 & 0.00 & 0.001 \\
\hline Nitrógeno amoniacal & $\mathrm{mg} / \mathrm{l}$ & 27.00 & & 0.32 & & 0.58 & \\
\hline
\end{tabular}


Anexo 2. Caracterización edáfica de las poblaciones naturales de camu camu

\begin{tabular}{|c|c|c|c|c|c|c|c|c|c|}
\hline \multirow[t]{3}{*}{ Carácter } & \multirow[t]{3}{*}{ Profundidad } & \multicolumn{8}{|c|}{ Población } \\
\hline & & \multicolumn{2}{|c|}{ Supay } & \multicolumn{2}{|c|}{ Sahua } & \multicolumn{2}{|c|}{ Palmichal } & \multicolumn{2}{|c|}{ Tapaje } \\
\hline & & 1999 & 2000 & 1999 & 2000 & 1999 & 2000 & 1999 & 2000 \\
\hline \multirow[t]{2}{*}{$\%$ de arena } & $0.00-0.15$ & 0 & 0.00 & 70 & 1.00 & 2 & 2.00 & 1 & 1.00 \\
\hline & $0.16-0.30$ & 0 & 0.00 & 72 & 0.00 & 0 & 2.00 & 1 & 0,00 \\
\hline \multirow[t]{2}{*}{$\%$ de limo } & $0.00-0.15$ & 8 & 7.00 & 10 & 59.00 & 6 & 6.00 & 3 & 2.00 \\
\hline & $0.16-0.30$ & 5 & 5.00 & 8 & 40.00 & 1 & 2.00 & 2 & 2.00 \\
\hline \multirow[t]{2}{*}{$\%$ de arcilla } & $0.00-0.15$ & 92 & 93.00 & 20 & 40.00 & 92 & 92.00 & 96 & 97.00 \\
\hline & $0.16-0.30$ & 95 & 95.00 & 20 & 60.00 & 99 & 96.00 & 97 & 98.00 \\
\hline \multirow[t]{2}{*}{$\mathrm{pH}$ (Unid/Stan) } & $0.00-0.15$ & 3.25 & 4.38 & 4.02 & 4.26 & 4.66 & 4.42 & 4.50 & 4.67 \\
\hline & $0.16-0.30$ & 3.98 & 4.08 & 3.97 & 4.66 & 4.04 & 4.56 & 4.29 & 4.40 \\
\hline \multirow[t]{2}{*}{ N (\%) } & $0.00-0.15$ & 0.19 & 0.40 & 0.20 & 0.40 & 0.45 & 0.50 & 0.20 & 0.50 \\
\hline & $0.16-0.30$ & 0.18 & 0.30 & 0.14 & 0.30 & 0.10 & 0.40 & 0.18 & 0.50 \\
\hline \multirow[t]{2}{*}{ M.O (\%) } & $0.00-0.15$ & 4.59 & 4.00 & 3.78 & 3.27 & 6.30 & 5.70 & 3.93 & 6.07 \\
\hline & $0.16-0.30$ & 2.71 & 1.83 & 2.63 & 1.86 & 2.79 & 2.94 & 3.27 & 2.47 \\
\hline \multirow[t]{2}{*}{$\mathrm{P}(\mathrm{ppm})$} & $0.00-0.15$ & 28.00 & 29.22 & 46.00 & 134.00 & 17.00 & 51.00 & 30.00 & 55.00 \\
\hline & $0.16-0.30$ & 21.00 & 142.00 & 30.00 & 136.00 & 9.00 & 54.00 & 26.00 & 98.00 \\
\hline \multirow[t]{2}{*}{$\mathrm{K}_{2} \mathrm{O}(\mathrm{Kg} / \mathrm{Ha})$} & $0.00-0.15$ & 1220.65 & 585.69 & 1071.53 & 1077.53 & 1444.34 & 944.34 & 1934.08 & 564.0 \\
\hline & $0.16-0.30$ & 1496.57 & 745.97 & 1092.53 & 585.69 & 1559.99 & 966.51 & 3832.84 & 609.79 \\
\hline \multirow[t]{2}{*}{$\mathrm{Ca}$ (meq/100 gr) } & $0.00-0.15$ & 5.15 & 9.86 & 4.05 & 11.60 & 1.24 & 7.25 & 1.24 & 6.87 \\
\hline & $0.16-0.30$ & 5.39 & 9.28 & 4.60 & 11.01 & 0.66 & 5.99 & 0.66 & 7.25 \\
\hline \multirow[t]{2}{*}{$\mathrm{Mg}(\mathrm{meq} / 100 \mathrm{~g})$} & $0.00-0.15$ & 3.74 & 7.15 & 2.94 & 8.40 & 0.70 & 5.25 & 0.70 & 4.98 \\
\hline & $0.16-0.30$ & 3.90 & 6.72 & 3.36 & 7.99 & 0.36 & 4.34 & 0.36 & 5.25 \\
\hline \multirow[t]{2}{*}{$\mathrm{K}(\mathrm{kg} / \mathrm{ha})$} & $0.00-0.15$ & 506.44 & 292.85 & 444.57 & 444.57 & 391.8 & 391.8 & 806.0 & 234.0 \\
\hline & $0.16-0.30$ & 620.92 & 309.50 & 453.29 & 292.85 & 647.23 & 401.0 & 1252.0 & 253.0 \\
\hline
\end{tabular}

Anexo 3. Inventario poblacional de rodales naturales de camu camu, años 1999 y 2000

\begin{tabular}{|c|c|c|c|c|c|c|c|c|c|c|c|c|}
\hline \multirow[t]{2}{*}{ Base diamétrica } & \multicolumn{3}{|c|}{ Supay } & \multicolumn{3}{|c|}{ Sahua } & \multicolumn{3}{|c|}{ Palmichal } & \multicolumn{3}{|c|}{ Tapaje } \\
\hline & 1999 & 2000 & Promedio & 1999 & 2000 & Promedio & 1999 & 2000 & Promedio & 1999 & 2000 & Promedio \\
\hline De $0.01 \mathrm{~cm} \mathrm{a} 1.99 \mathrm{~cm}$ & 61 & 31 & 46 & 82 & 22 & 52 & 113 & 111 & 112 & 134 & 118 & 126 \\
\hline De $2.00 \mathrm{~cm}$ a 2. & 87 & 45 & 66 & 104 & 32 & 68 & 149 & 131 & 140 & 152 & 134 & 143 \\
\hline De $3.00 \mathrm{~cm}$ a 3. & 76 & 40 & 58 & 147 & 42 & 94 & 109 & 94 & 101 & 152 & 134 & 143 \\
\hline De $4.00 \mathrm{cr}$ & 69 & 34 & 5 & 131 & & & 20 & 20 & 20 & 62 & 56 & 59 \\
\hline Mayores de $5 \mathrm{~cm}$ & 76 & 39 & 57 & 82 & 23 & 53 & 8 & 2 & 5 & 10 & 8 & 9 \\
\hline TOTAL & 368 & 189 & 278 & 546 & 154 & 350 & 399 & 356 & 377 & 509 & 417 & 463 \\
\hline Densidad (ha) & 36750 & 18850 & 27800 & 54550 & 15400 & 34975 & 39,850 & 35600 & 37725 & 50850 & 41700 & 46275 \\
\hline Especies atípicas * & 1400 & 3913 & 2657 & 1100 & 6600 & 3850 & 19950 & 4750 & 12350 & 5750 & 4750 & 5250 \\
\hline$\%$ camu camu & 96.34 & 73.01 & 91.00 & 97.67 & 59.48 & 90.08 & 66.65 & 88.92 & 75.34 & 90.39 & 88.92 & 89.81 \\
\hline
\end{tabular}

* Especies diferentes al camu camu 
Anexo 4. Producción de poblaciones naturales de camu camu, 1999 kg

\begin{tabular}{|c|c|c|c|c|c|c|c|c|}
\hline \multirow[t]{2}{*}{ Base diamétrica } & \multicolumn{2}{|c|}{ Supay $100 \mathrm{~m}^{2}$} & \multicolumn{2}{|c|}{ Sahua $100 \mathrm{~m}^{2}$} & \multicolumn{2}{|c|}{ Palmichal $100 \mathrm{~m}^{2}$} & \multicolumn{2}{|c|}{ Tapaje $100 \mathrm{~m}^{2}$} \\
\hline & Parcela 1 & Parcela 2 & Parcela 1 & Parcela 2 & Parcela 1 & Parcela 2 & Parcela 1 & Parcela 2 \\
\hline De $0.01 \mathrm{~cm}$ a $1.99 \mathrm{~cm}$ & 10 & 14 & 11 & 7 & 22 & 18 & 21 & 20 \\
\hline De $2.00 . \mathrm{cm}$ a $2.99 \mathrm{~cm}$ & 37 & 33 & 36 & 34 & 56 & 58 & 52 & 55 \\
\hline De $3.00 \mathrm{~cm}$ a $3.99 \mathrm{~cm}$ & 32 & 54 & 51 & 55 & 47 & 44 & 52 & 47 \\
\hline De $4.00 \mathrm{~cm}$ a $4.99 \mathrm{~cm}$ & 28 & 41 & 46 & 54 & 9 & 8 & 21 & 13 \\
\hline Mayores de $5 \mathrm{~cm}$ & 30 & 35 & 29 & 26 & 3 & 3 & 3 & 3 \\
\hline TOTAL & 137 & 177 & 173 & 176 & 137 & 131 & 148 & 139 \\
\hline $\mathrm{Kg} / \mathrm{ha}$ & 13700 & 17700 & 17300 & 17600 & 13700 & 13100 & 14800 & 13900 \\
\hline Promedio kg/ha & \multicolumn{2}{|c|}{15700} & \multicolumn{2}{|c|}{17450} & \multicolumn{2}{|c|}{13400} & \multicolumn{2}{|c|}{14350} \\
\hline
\end{tabular}

Anexo 5. Producción de poblaciones de camu camu, $2000 \mathrm{~kg}$

\begin{tabular}{|c|c|c|c|c|c|c|c|c|}
\hline \multirow[t]{2}{*}{ Base diamétrica } & \multicolumn{2}{|c|}{ Supay $100 \mathrm{~m}^{2}$} & \multicolumn{2}{|c|}{ Sahua $100 \mathrm{~m}^{2}$} & \multicolumn{2}{|c|}{ Palmichal $100 \mathrm{~m}^{2}$} & \multicolumn{2}{|c|}{ Tapaje $100 \mathrm{~m}^{2}$} \\
\hline & Parcela 1 & Parcela 2 & Parcela 1 & Parcela 2 & Parcela 1 & Parcela 2 & Parcela 1 & Parcela 2 \\
\hline De $0.01 \mathrm{~cm} \mathrm{a} 1.99 \mathrm{~cm}$ & 6.5 & 8.5 & 5.5 & 5.5 & 23 & 23.5 & 23.5 & 18.5 \\
\hline De $2.00 . \mathrm{cm} \mathrm{a} 2.99 \mathrm{~cm}$ & 11.5 & 26.5 & 7.25 & 19.5 & 48.5 & 48 & 35.5 & 37 \\
\hline De $3.00 \mathrm{~cm} \mathrm{a} 3.99 \mathrm{~cm}$ & 14 & 32.5 & 24 & 28.5 & 50.5 & 54 & 49.5 & 53 \\
\hline De $4.00 \mathrm{~cm} \mathrm{a} 4.99 \mathrm{~cm}$ & 14 & 37.5 & 23.75 & 28 & 13 & 7 & 27 & 29 \\
\hline Mayores de $5 \mathrm{~cm}$ & 22 & 50 & 12.75 & 26.5 & 2 & 1 & 1 & 1 \\
\hline TOTAL & 67.5 & 155.5 & 73.75 & 108 & 137 & 133.5 & 125 & 138.5 \\
\hline $\mathrm{Kg} / \mathrm{ha}$ & 6750 & 15550 & 7375 & 10800 & 13700 & 13350 & 12500 & 13850 \\
\hline Promedio kg/ha & \multicolumn{2}{|c|}{11150} & \multicolumn{2}{|c|}{9125} & \multicolumn{2}{|c|}{13525} & \multicolumn{2}{|c|}{13750} \\
\hline
\end{tabular}

Anexo 6. Biometría de frutos de las poblaciones de camu camu

\begin{tabular}{lrrrrrrrr}
\hline & Largo & \multicolumn{1}{c}{ Ancho } & Peso g & Número & Peso g & Peso g & Peso g & \multicolumn{1}{c}{$\%$} \\
\hline Poblaciones & Fruto cm & Fruto cm & Fruto & Semillas & Semillas & Cáscara & Pulpa & Pulpa \\
Supay Cocha & 2.33 & 2.51 & 7.99 & 2.96 & 1.56 & 1.55 & 4.88 & 60.12 \\
Sahua Cocha & 2.37 & 2.53 & 8.63 & 2.78 & 1.58 & 1.58 & 5.37 & 62.15 \\
Palmichal & 2.08 & 2.20 & 5.95 & 2.93 & 1.29 & 1.40 & 3.26 & 54.31 \\
Tapaje & 2.34 & 2.49 & 8.27 & 3.00 & 1.44 & 1.54 & 5.28 & 63.86 \\
Máximo & 2.37 & 2.53 & 8.63 & 3.00 & 1.58 & 1.58 & 5.37 & 63.86 \\
Promedio & $\mathbf{2 . 2 8}$ & $\mathbf{2 . 4 3}$ & $\mathbf{7 . 7 1}$ & $\mathbf{2 . 9 2}$ & $\mathbf{1 . 4 7}$ & $\mathbf{1 . 5 2}$ & $\mathbf{4 . 7 0}$ & $\mathbf{6 0 . 1 1}$ \\
Mínimo & 2.08 & 2.20 & 5.95 & 2.78 & 1.29 & 1.40 & 3.26 & 54.31 \\
DesVest & 0.13 & 0.16 & 1.20 & 0.10 & 0.13 & 0.08 & 0.98 & 4.16 \\
CoeVar & 5.90 & 6.41 & 15.59 & 3.29 & 9.10 & 5.28 & 20.90 & 6.92 \\
\hline
\end{tabular}


Anexo 7. Especies atípicas que se encuentran en las poblaciones naturales de camu camu

\begin{tabular}{|c|c|c|c|c|c|}
\hline \multicolumn{2}{|l|}{ Especie } & \multirow{2}{*}{$\begin{array}{c}\begin{array}{c}\text { Supay } \\
\text { Cocha }\end{array} \\
\text { Individuos/ha }\end{array}$} & \multirow{2}{*}{$\begin{array}{c}\begin{array}{c}\text { Sahua } \\
\text { Cocha }\end{array} \\
\text { Individuos/ha }\end{array}$} & \multirow{2}{*}{$\begin{array}{l}\text { Palmichal } \\
\text { Individuos/ha }\end{array}$} & \multirow{2}{*}{$\begin{array}{c}\text { Tapaje } \\
\text { Individuos/ha }\end{array}$} \\
\hline Nombre científico & Nombre vulgar & & & & \\
\hline Eugenia inundata & Fanache & 2033 & 1925 & & \\
\hline Cecropia cetico & Cetico & 156 & & & \\
\hline Acacia sp. & Acacia & 156 & 231 & & \\
\hline Machaerium floribundum & Garabato casha & 156 & & & \\
\hline Xylosma intermedium & Limón casha & 156 & 732 & & \\
\hline Coccoloba barbeyana & Cocoloba & & 962 & & \\
\hline Mabea nitida & Sabalohuayo & & & 11250 & 2100 \\
\hline Stylogyne longifolia & Acarahuazú panga & & & 100 & 3045 \\
\hline Crateva tapia tamara & Tamara & & & & 52 \\
\hline Macrolobium acaciifolium & Irapari, pashaco & & & & 53 \\
\hline
\end{tabular}

